dish bottoms. A thermometer is attached to the glass plate to provide temperature references. A powerstat utilized with the heat gun prevents off-on heat surges and when set at 85-90 V gives satisfactory heated air volume at the 8.5-9 in. distance at laboratory temperatures of $25^{\circ}-26^{\circ} \mathrm{C}$. Laboratory temperatures less than $25^{\circ} \mathrm{C}$ will require one or more of the following adjustments: (1) increased powerstat settings; (2) partial closure of heat gun air intake vents; (3) shortened distance between deflector and stage.

The final thermostat setting should be made only after the microscope, which plays an integral part in temperature maintenanco, is equilibrated to the desired temperature. To do this the unit should be turned on at the selected powerstat setting and allowed to operate for $1 \mathrm{~h}$ while the thermostat setting is adjusted ito maintain the desired stage temperature. This procedure will prevent croeping overheating of the stage. Regular checks of stage temporature should be used while observing cultures.

It is suggested that the unit be pre-heated for $1 \mathrm{~h}$ prior to placing cultures on the stage and that the unit be shut down temporarily while photographs are being taken in order to obviate vibration if long exposures are required. Such short off-periods do not disturb temperature equilibration.

Laboratory of Histology and Embryology, University of Pennsylvania Dental School, Philadelphia 4.

\section{Calculation of Exposure Times for Successive Autoradiographs}

AUTORADIOGRAPHIC investigations often require very large numbers of exposures. It is, therefore, necessary to have a rapid method for calculating the exposure timos for the successive specimens of a series. Stowell ${ }^{1}$, in a communication directing attention to the value of special purpose slide rules, has described one on which the calculation may be made very rapidly.

The calculation is necessary because a radioactive isotope may lose a large part of its activity in the time taken to prepare the later specimens. For constant exposure (that is, radiation dose), the exposure times used must satisfy the equation:

$$
1-e^{-\lambda t_{s}}=\theta^{-\lambda t_{3}}-e^{-\lambda t_{3}}
$$

where the zero of time is taken at the start of the first, or standard, exposure which ends at $\operatorname{tim} \theta t_{8}$, and any other exposure beginning at $t_{1}$ ends at $t_{2} . \quad \lambda$ is equal to 0.693 divided by the isotope half-life.

Stowell's rule has a time-scale, calibrated according to the function $\left(1-e^{-\lambda t}\right)$, and a cursor bearing two lines $\left(1-e^{\left.-\lambda t_{s}\right)}\right.$ apart. The values of $\lambda$ and $t_{s}$ are fixed once the rule has been calibrated and, although several scales might be included on the rule, it cannot be considered as a general purpose instrument in this field. The time-scale is calibrated in clock time so that it is easy to find exposures that begin and end during working hours. There is, however, the disadvantage that the time zero is thereby fixed at one time of the day.

The best solution to this problem would be a calculator that required no further modification to cover all possible calculations of this type. A graph showing exposure time $\left(t_{2}-t_{1}\right)$ as a function of delay time $\left(t_{1}\right)$ appears to be the simplest method of presenting the data. A single curve will cover all possibilities if the time delay scale is movable so that its zero can be put at the point where the exposure time is equal to $t_{s}$. Unfortunately, this is only possible with a linear scale; the same result could be obtained with other scales by adding a constant to all delay time readings, but this would be very inconvenient. For constant reading accuracy logarithmic scales are necessary, so that a family of curves must be plotted with different values of $t_{s}$. A set of such curves is shown in Fig. 1, where the value of $t_{s}$ for each curve is equal to the asymptotic value of $\left(t_{2}-t_{1}\right)$ as $t_{1}$ tends to zero. The lowest curve gives 80 per cent of the doso given by the next curve and this is the largest difference for the set shown. It will usually be possible to use one of the curves, but for intermediate values of $t_{s}$ the errors involved in interpolation can normally be considered insignificant.

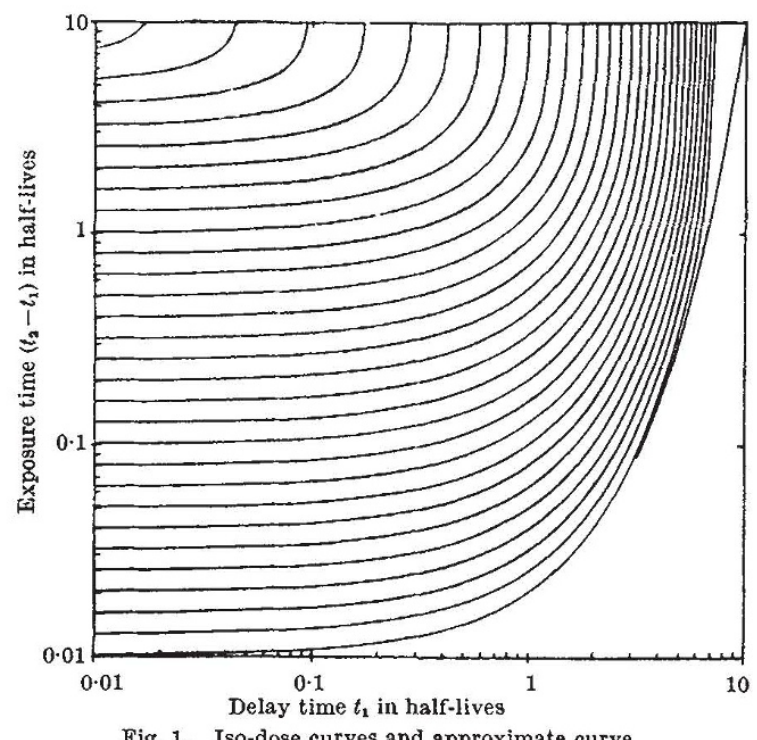

Since the curves are to be used for isotopes with various half-life times, the scalos are calibrated directly in one half-life units and it is necessary to multiply the scale readings by the half-life of the isotope in use. The multiplication may be avoided with the aid of a transparent, two-dimensional, logarithmic graticule, with decade widths equal to those of the graph (for example, a sheet of log-log graph tracing paper). The graticule may be calibrated in any convenient time units, and is placed over the graph so that the one half-life co-ordinates of the graph are aligned exactly with the co-ordinates of appropriate value on the graticule. The graph may then be read directly on the graticule in whatever time units have been chosen.

Extension of the curves in any direction except to smaller values of $t_{s}$ seems unnecessary. For all exposures less than 0.028 half-life the curves lie within 1 per cent of the value given by:

$$
t_{2}-t_{1}=t_{8} \mathrm{e}^{\lambda t_{1}}
$$

This function, with $t_{s}=0.01$ half-life, is shown on the right in the figure, in the region where it differs from the lowest of the exact curves by more than 3 per cent. Thus, for shorter exposures, this curve may be used simply by displacing it downwards to the required extent, without any loss of accuracy. An analysis has been made of the errors involved in the use of the approximate equation. The results suggest a further approach to the problem, which is being prepared for publication.

The only disadvantage of this calculator appears to be that the times read from it will not be actual clock times but time durations. There is, however, no restriction on the time zero for the experiments.

I thank Prof. R. A. Buckingham and the staff of the University of London Computer Unit for their help in the calculation of the data for the graph.

\section{Institute of Laryngology and Otology} (University of London),

330/332 Gray's Inn Road, London, W.C.1.

- Present address: The Biological Laboratory, Harvard University, Cambridge, 38, Mass.

${ }^{1}$ Stowell, E. C., Nature, 198, 318 (1963). 